\title{
TOEPLITZ OPERATORS ON HARMONIC BERGMAN SPACES
}

\author{
BOO RIM CHOE, YOUNG JOO LEE AND KYUNGUK NA
}

\begin{abstract}
We study Toeplitz operators on the harmonic Bergman spaces on bounded smooth domains. Two classes of symbols are considered; one is the class of positive symbols and the other is the class of uniformly continuous symbols. For positive symbols, boundedness, compactness, and membership in the Schatten classes are characterized. For uniformly continuous symbols, the essential spectra are described.
\end{abstract}

\section{$\S 1$. Introduction}

Throughout the paper, $\Omega \subset \mathbb{R}^{n}(n \geq 2)$ denotes a fixed bounded domain with $C^{\infty}$-boundary. For $1 \leq p<\infty$, the harmonic Bergman space $b^{p}=$ $b^{p}(\Omega)$ is the set of all complex-valued harmonic functions $f$ on $\Omega$ for which

$$
\|f\|_{p}=\left\{\int_{\Omega}|f|^{p} d V\right\}^{1 / p}<\infty .
$$

Here and elsewhere, $V$ denotes the Lebesgue volume measure on $\Omega$. We will also use the notation $d y=d V(y)$ for simplicity. Also, we let $b^{\infty}$ denote the space of all bounded harmonic functions on $\Omega$.

As is well known, $b^{p}$ is a closed subspace of $L^{p}=L^{p}(\Omega, V)$ and hence a Banach space. In particular, $b^{2}$ is a Hilbert space. Each point evaluation is easily verified to be a bounded linear functional on $b^{2}$. Hence, for each $x \in \Omega$, there exists a unique function $R(x, \cdot) \in b^{2}$ which has the following reproducing property:

$$
f(x)=\int_{\Omega} f(y) \overline{R(x, y)} d y \quad(x \in \Omega)
$$

for all $f \in b^{2}(\Omega)$.

Let $Q$ be the Hilbert space orthogonal projection from $L^{2}$ onto $b^{2}$. The reproducing kernels $R(x, \cdot)$ are known to be symmetric and real-valued. See

Received July 22, 2002.

2000 Mathematics Subject Classification: Primary 47B35, Secondary 31 B05.

This work is supported by the Korea Research Foundation Grant(KRF-2000DP0014). 
[1] for more information and related facts. Hence, the reproducing property mentioned above yields the following integral representation of $Q$ :

$$
Q \psi(x)=\int_{\Omega} \psi(y) R(x, y) d y \quad(x \in \Omega)
$$

for functions $\psi \in L^{2}$. For each fixed $x \in \Omega$, the function $R(x, \cdot)$ is known to be bounded on $\Omega$ (see Lemma 2.1). Thus, the projection $Q$ naturally extends to an integral operator via (1.1) from $L^{1}$ into the space of all harmonic functions on $\Omega$. Moreover, for $1<p<\infty$, it is known that $Q$ is a bounded projection from $L^{p}$ onto $b^{p}$. See Theorem 4.2 of [5]. Using this $L^{p}$-boundedness of $Q$, one can see that $b^{\infty}$ is dense in each of $b^{p}, 1<p<\infty$ (see Lemma 2.5).

The integral transform $Q$ even extends to $M(\Omega)$, the space of all complex Borel measures on $\Omega$. Namely, for each $\mu \in M(\Omega)$, the integral

$$
Q \mu(x)=\int_{\Omega} R(x, y) d \mu(y) \quad(x \in \Omega)
$$

defines a function harmonic on $\Omega$ (harmonicity can be checked by using Lemma 2.1 below).

For $\mu \in M(\Omega)$, the Toeplitz operator $T_{\mu}$ with symbol $\mu$ is defined by

$$
T_{\mu} f=Q(f d \mu)
$$

for $f \in b^{\infty}$. In case $\mu=\varphi d V$, we will write $T_{\mu}=T_{\varphi}$. Note that $T_{\mu}$ is densely defined on $b^{p}$ for each $1<p<\infty$.

In this paper, we consider two classes of symbols. One is the class of positive symbols and the other is the class of uniformly continuous symbols. For positive symbols, we give characterizations for corresponding Toeplitz operators to be bounded, compact and in the Schatten classes. For uniformly continuous symbols, we find the essential spectra of corresponding Toeplitz operators. Previously, Toeplitz operators with positive or uniformly continuous symbols were studied in [4], [6], [8] on the ball; see [2] for results on the half-space. Our results show that previously known results continue to hold for general bounded smooth domains.

In Section 2 several preliminary facts are collected. Section 3 is devoted to results concerning positive symbols. Section 4 is devoted to results concerning uniformly continuous symbols.

Constants. We will use the same letter $C$ to denote various positive constants, often with subscripts indicating dependency, which may change at each occurrence. We will often abbreviate inessential constants involved in inequalities by writing $A \lesssim B$ for positive quantities $A$ and $B$ if the ratio $A / B$ has a positive upper bound. Also, we write $A \approx B$ if $A \lesssim B$ and $B \lesssim A$ 


\section{§2. Basic Lemmas}

The main ingredients to our work in this paper are fundamental estimates obtained by Kang and Koo [5]. So, in this section, we recall some results in [5] and prove some basic consequences.

We let $r(x)=\operatorname{dist}(x, \partial \Omega)$ for $x \in \Omega$. Also, for $x, y \in \Omega$, we define

$$
d(x, y)=|x-y|+r(x)+r(y) .
$$

Note that our definition of $r$ is slightly different from that in [5]. In [5] the definition of $r$ requires some smoothness off the boundary of $\Omega$. However, as far as estimates in Lemma 2.1 and Lemma 2.2 are concerned, those two definitions does not make any difference.

We first recall a couple of results in [5]. The first one is the size estimates of derivatives of the reproducing kernels. In the following we use the conventional multi-index notations. That is, for an ordered $n$-tuple $\alpha=\left(\alpha_{1}, \cdots, \alpha_{n}\right)$ of nonnegative integers, we let $|\alpha|=\alpha_{1}+\cdots+\alpha_{n}$ and $\partial_{x}^{\alpha}=\left(\partial / \partial x_{1}\right)^{\alpha_{1}} \cdots\left(\partial / \partial x_{n}\right)^{\alpha_{n}}$.

LEMMA 2.1. Given multi-indices $\alpha$ and $\beta$, there exists a constant $C_{\alpha, \beta}$ such that

$$
\left|\partial_{x}^{\alpha} \partial_{y}^{\beta} R(x, y)\right| \leq \frac{C_{\alpha, \beta}}{d(x, y)^{n+|\alpha|+|\beta|}}
$$

for $x, y \in \Omega$. Moreover, there exists a constant $C$ such that

$$
R(x, x) \geq \frac{C}{r(x)^{n}}
$$

for $x \in \Omega$.

Proof. See Theorem 1.1 of [5].

The second one is the integral estimates which, together with Lemma 2.1, enable us to estimate integral behavior of the reproducing kernels.

LEMMA 2.2. For $s, t \geq 0$ with $s+t>0$ and $t<1$, there exists a constant $C_{s, t}$ such that

$$
\int_{\Omega} \frac{d y}{d(x, y)^{n+s} r(y)^{t}} \leq \frac{C_{s, t}}{r(x)^{s+t}}
$$

for $x \in \Omega$.

Proof. See Lemma 4.1 of [5]. 
Our first observation is that the estimate (2.2) remains valid for points $x, y$ sufficiently close to each other. To be more precise, let

$$
E_{\delta}(x)=\{y \in \Omega:|x-y|<\delta r(x)\}
$$

for $0<\delta<1$ and $x \in \Omega$. Since $\delta<1$, we note that $E_{\delta}(x)$ is the euclidean ball with center at $x$ and radius $\delta r(x)$. We then have the following.

Lemma 2.3. There exist some $\delta_{0} \in(0,1)$ and a constant $C$ such that

$$
C^{-1} \leq R(x, y) r(x)^{n} \leq C
$$

whenever $x \in \Omega$ and $y \in E_{\delta_{0}}(x)$.

Proof. Let $x \in \Omega$ and $y \in E_{\delta}(x)$ for $\delta$ to be chosen later. Then, we have by $(2.1)$

$$
\begin{aligned}
R(x, x)-R(x, y) & \leq \sup _{z \in E_{\delta}(x)}\left|\nabla_{z} R(x, z)\right||x-y| \\
& \lesssim \frac{\delta r(x)}{d(x, z)^{n+1}} \\
& \leq \frac{\delta}{r(x)^{n}}
\end{aligned}
$$

where $\nabla_{z}$ denotes the gradient with respect to $z$-variable. It follows from (2.2) that

$$
R(x, y) \geq R(x, x)-\frac{O(\delta)}{r(x)^{n}} \gtrsim \frac{1-O(\delta)}{r(x)^{n}} .
$$

Now, taking $\delta$ small enough, we have

$$
R(x, y) \gtrsim \frac{1}{r(x)^{n}} .
$$

This shows the lower estimate. The upper estimate is a consequence of (2.1). The proof is complete.

Next, we estimate the $L^{p}$-norms of the reproducing kernels.

LemmA 2.4. Let $1<p<\infty$. Then there is a constant $C_{p}$ such that

$$
C_{p}^{-1} \leq\|R(x, \cdot)\|_{p} r(x)^{n\left(1-\frac{1}{p}\right)} \leq C_{p}
$$

for every $x \in \Omega$. 
Proof. Let $x \in \Omega$. It follows from Lemma 2.1 and Lemma 2.2 that

$$
\|R(x, \cdot)\|_{p}^{p} \lesssim \int_{\Omega} \frac{1}{d(x, y)^{n p}} d y \lesssim \frac{1}{r(x)^{n(p-1)}} .
$$

For the converse inequality, fix $\delta_{0}$ provided by Lemma 2.3. Then, we have

$$
\|R(x, \cdot)\|_{p}^{p} \geq \int_{E_{\delta_{0}}(x)}|R(x, y)|^{p} d y \approx \frac{V\left(E_{\delta_{0}}(x)\right)}{r(x)^{n p}} \approx \frac{1}{r(x)^{n(p-1)}} .
$$

The proof is complete.

Next, we prove the density of $b^{\infty}$ in $b^{p}$. We will use the $L^{p}$-boundedness of the projection $Q$ for $1<p<\infty$, which is mentioned in the introduction.

Lemma 2.5. The space $b^{\infty}$ is dense in $b^{p}$ for each $1<p<\infty$.

Proof. Let $1<p<\infty$ and $f \in b^{p}$. For $\epsilon>0$, we let $f_{\epsilon}=f \chi_{\Omega_{\epsilon}}$ where $\Omega_{\epsilon}=\{x \in \Omega: r(x) \geq \epsilon\}$. By Lemma 2.1, one can see $Q f_{\epsilon} \in b^{\infty}$ for each $\epsilon$. Moreover, by boundedness of $Q$, we have

$$
\left\|f-Q f_{\epsilon}\right\|_{p}=\left\|Q\left(f-f_{\epsilon}\right)\right\|_{p} \lesssim\left\|f-f_{\epsilon}\right\|_{p}
$$

for each $\epsilon$. Since the last term above goes to 0 as $\epsilon \rightarrow 0$, we have the desired result. The proof is complete.

We remark in passing that Lemma 2.5 remains valid for $p=1$. This can be seen by constructing nonorthogonal $L^{1}$-bounded projections, which were not considered in [5]. Finally, we need the dualities. Given $1<p<\infty$, the exponent $p^{\prime}$ will always denote the conjugate exponent of $p$, i.e., $1 / p+1 / p^{\prime}=1$.

Lemma 2.6. Let $1<p<\infty$. Then the dual of $b^{p}$ is $b^{p^{\prime}}$ under the usual pairing

$$
\langle f, g\rangle=\int_{\Omega} f \bar{g} d V .
$$

Proof. See Corollary 4.3 of [5].

\section{§3. Positive Toeplitz operators}

In this section, we consider positive symbols and give characterizations for corresponding Toeplitz operators to be bounded, compact and in the Schatten classes. Our characterizations will be in terms of Carleson measures. 
Let $1 \leq p<\infty$. Given a finite positive Borel measure $\mu$ on $\Omega$ (we will simply write $\mu \geq 0$ ), we say that $\mu$ is a Carleson measure on $b^{p}$ if there exists a constant $C>0$ such that

$$
\int_{\Omega}|f|^{p} d \mu \leq C \int_{\Omega}|f|^{p} d V
$$

for all $f \in b^{p}$. So, $\mu$ is a Carleson measure on $b^{p}$ if and only if the inclusion $i_{p}: b^{p} \rightarrow L^{p}(\mu)$ is bounded.

In order to describe Carleson measures on $b^{p}$, we need notions of averaging functions and Berezin transforms. Let $\mu \geq 0$. For $\delta \in(0,1)$, the averaging function $\widehat{\mu}_{\delta}$ of $\mu$ over the balls $E_{\delta}(x)$ is defined by

$$
\widehat{\mu}_{\delta}(x)=\frac{\mu\left(E_{\delta}(x)\right)}{V\left(E_{\delta}(x)\right)} \quad(x \in \Omega) .
$$

Also, for $1<p<\infty$, we define the Berezin $p$-transform $\tilde{\mu}_{p}$ on $\Omega$ by

$$
\tilde{\mu}_{p}(a)=\int_{\Omega}\left|k_{a, p}\right|^{p} d \mu \quad(a \in \Omega)
$$

where

$$
k_{a, p}(x)=\frac{R(x, a)}{\|R(\cdot, a)\|_{p}} \quad(x \in \Omega)
$$

is the $L^{p}$-normalized reproducing kernel.

As is the case on various other settings, Carleson measures will be characterized in terms of averaging functions and Berezin transforms.

LEMma 3.1. Let $\delta \in(0,1)$. Then we have

$$
(1-\delta) r(x)<r(y)<(1+\delta) r(x)
$$

for $x \in \Omega$ and $y \in E_{\delta}(x)$.

Proof. Let $x \in \Omega$ and $y \in E_{\delta}(x)$. Then, for any $\zeta \in \partial \Omega$, we have

$$
r(y) \leq|y-\zeta| \leq|x-y|+|x-\zeta|<\delta r(x)+|x-\zeta| .
$$

Hence, taking the infimum over all $\zeta \in \partial \Omega$, we have the second inequality. The first inequality can be verified similarly. The proof is complete.

LEMmA 3.2. Let $\delta, \epsilon \in(0,1)$. Then, there exist constants $C_{\delta, \epsilon}$ such that the following hold for all $\mu \geq 0$ and $x \in \Omega$.

(a) $\mu\left(E_{\delta}(x)\right) \leq \frac{C_{\delta, \epsilon}}{V\left(E_{\delta}(x)\right)} \int_{E_{\delta}(x)} \mu\left(E_{\epsilon}(y)\right) d y$. 
(b) $\widehat{\mu}_{\delta}(x) \leq \frac{C_{\delta, \epsilon}}{V\left(E_{\delta}(x)\right)} \int_{E_{\delta}(x)} \widehat{\mu}_{\epsilon}(y) d y$.

Proof. Let $\mu \geq 0$ and $x \in \Omega$. Note that if $y \in E_{\frac{\epsilon}{1+\epsilon}}(w)$, then $w \in E_{\epsilon}(y)$ by Lemma 3.1. In other words, we have

$$
\chi_{E_{\frac{\epsilon}{1+\epsilon}}}(w)(y) \leq \chi_{E_{\epsilon}(y)}(w)
$$

for $w, y \in \Omega$. Here, $\chi_{E}$ denotes the characteristic function of $E$. It follows that

$$
\begin{aligned}
\int_{E_{\delta}(x)} \mu\left(E_{\epsilon}(y)\right) d y & =\int_{E_{\delta}(x)} \int_{E_{\epsilon}(y)} d \mu(w) d y \\
& =\int_{\Omega} \int_{E_{\delta}(x)} \chi_{E_{\epsilon}(y)}(w) d y d \mu(w) \\
& \geq \int_{E_{\delta}(x)} \int_{E_{\delta}(x)} \chi_{E_{\frac{\epsilon}{1+\epsilon}}(w)}(y) d y d \mu(w) \\
& =\int_{E_{\delta}(x)} V\left[E_{\delta}(x) \cap E_{\frac{\epsilon}{1+\epsilon}}(w)\right] d \mu(w) \\
& \geq \mu\left(E_{\delta}(x)\right) \inf _{w \in E_{\delta}(x)} V\left[E_{\delta}(x) \cap E_{\frac{\epsilon}{1+\epsilon}}(w)\right] .
\end{aligned}
$$

Thus, in order to prove (a), it remains to show

$$
\inf _{w \in E_{\delta}(x)} V\left[E_{\delta}(x) \cap E_{\frac{\epsilon}{1+\epsilon}}(w)\right] \gtrsim r(x)^{n} .
$$

To see this, let $w \in E_{\delta}(x)$ and write $w=x+t \zeta$ where $0 \leq t<\delta r(x)$ and $|\zeta|=1$. If $0 \leq t<\frac{\epsilon}{1+\epsilon} r(w)-\delta r(x)$, then $E_{\delta}(x) \subset E_{\frac{\epsilon}{1+\epsilon}}(w)$. If $t>\frac{\epsilon}{1+\epsilon} r(w)-\delta r(x)$, then $E_{\delta}(x) \cap E_{\frac{\epsilon}{1+\epsilon}}(w)$ contains the ball with center at $w-\frac{\epsilon}{2(1+\epsilon)} r(w) \zeta$ and radius $\frac{\epsilon}{2(1+\epsilon)} r(w)$. Thus, by Lemma 3.1, we have (3.1) as desired. So, (a) holds. Also, (b) follows from (a) and Lemma 3.1. The proof is complete.

As an easy consequence of Lemma 3.2, we have the following.

Corollary 3.3. Let $\mu \geq 0$. If $\widehat{\mu}_{\epsilon}$ is bounded for some $\epsilon \in(0,1)$, then so is $\widehat{\mu}_{\delta}$ for all $\delta \in(0,1)$.

We also need a decomposition of $\Omega$ whose proof is essentially the same as that of Lemma of Covering in [7]. So, we omit the details.

Lemma 3.4. Let $\delta \in(0,1)$. Then, there exists a sequence $\left\{a_{i}\right\}$ in $\Omega$ satisfying the following two conditions:

(a) $\cup_{i=1}^{\infty} E_{\frac{\delta}{3}}\left(a_{i}\right)=\Omega$. 
(b) There is a positive integer $N$ such that each $E_{\delta}\left(a_{i}\right)$ intersects at most $N$ of the balls $E_{\delta}\left(a_{i}\right)$.

Note. In what follows, the sequence $\left\{a_{i}\right\}$ will always refer to the sequence chosen in Lemma 3.4. Note that $a_{i} \rightarrow \partial \Omega$ as $i \rightarrow \infty$.

Now, we characterize Carleson measures on $b^{p}$ in terms of averaging functions and Berezin transforms.

Theorem 3.5. Let $1<p<\infty$ and $\delta \in(0,1)$. For $\mu \geq 0$, the following conditions are all equivalent.

(a) $\mu$ is a Carleson measure on $b^{p}$.

(b) $\tilde{\mu}_{p}$ is bounded on $\Omega$.

(c) $\widehat{\mu}_{\delta}$ is bounded on $\Omega$.

(d) The sequence $\left\{\widehat{\mu}_{\delta}\left(a_{i}\right)\right\}$ is bounded.

Note that conditions (a) and (b) are independent of $\delta$, while conditions (c) and (d) are independent of $p$. Thus, the notion of Carleson measures on $b^{p}$ is independent of $1<p<\infty$. So, we will simply say that $\mu \geq 0$ is a Carleson measure if one of the four conditions above holds for $\mu$.

Proof. Since each $k_{a, p}$ has $L^{p}$-norm 1, the implication (a) $\Rightarrow$ (b) follows immediately.

Next, suppose (b) and show (c). To prove (c), we only need to consider $\delta$ sufficiently small by Corollary 3.3. So, assume $\delta=\delta_{0}$ where $\delta_{0}$ is the number provided by Lemma 2.3. Let $a \in \Omega$. By Lemma 2.3 and Lemma 2.4 , we have

$$
\int_{E_{\delta}(a)}\left|k_{a, p}\right|^{p} d \mu \approx r(a)^{n(p-1)} \int_{E_{\delta}(a)}|R(x, a)|^{p} d \mu(x) \approx \frac{r(a)^{n(p-1)}}{r(a)^{n p}} \mu\left(E_{\delta}(a)\right)
$$

so that

$$
\frac{\mu\left(E_{\delta}(a)\right)}{r(a)^{n}} \approx \int_{E_{\delta}(a)}\left|k_{a, p}\right|^{p} d \mu \leq \int_{\Omega}\left|k_{a, p}\right|^{p} d \mu \leq \sup _{a \in \Omega} \tilde{\mu}_{p}(a)<\infty .
$$

Now, since $V\left(E_{\delta}(a)\right) \approx r(a)^{n}$, we have $(\mathrm{c})$.

Clearly, we have $(\mathrm{c}) \Rightarrow(\mathrm{d})$.

Finally, suppose (d) and show (a). Let $f \in b^{p}$. First note that

$$
|f(x)|^{p} \lesssim \frac{1}{r(x)^{n}} \int_{E_{\delta / 3}(x)}|f|^{p} d V
$$


for $x \in \Omega$. This, together with Lemma 3.1, yields

$$
\begin{aligned}
\sup _{x \in E_{\delta / 3}(a)}|f(x)|^{p} & \lesssim \sup _{x \in E_{\delta / 3}(a)} \frac{1}{r(x)^{n}} \int_{E_{\delta / 3}(x)}|f|^{p} d V \\
& \lesssim \frac{1}{r(a)^{n}} \int_{E_{\delta}(a)}|f|^{p} d V \\
& \lesssim \frac{1}{V\left(E_{\delta}(a)\right)} \int_{E_{\delta}(a)}|f|^{p} d V
\end{aligned}
$$

for $a \in \Omega$. Now, by Lemma 3.4, we have

$$
\begin{aligned}
\int_{\Omega}|f|^{p} d \mu & \leq \sum_{i=1}^{\infty} \int_{E_{\delta / 3}\left(a_{i}\right)}|f|^{p} d \mu \\
& \leq \sum_{i=1}^{\infty} \mu\left(E_{\delta / 3}\left(a_{i}\right)\right) \sup _{x \in E_{\delta / 3}\left(a_{i}\right)}|f(x)|^{p} \\
& \lesssim \sum_{i=1}^{\infty} \frac{\mu\left(E_{\delta}\left(a_{i}\right)\right)}{V\left(E_{\delta}\left(a_{i}\right)\right)} \int_{E_{\delta}\left(a_{i}\right)}|f|^{p} d V \\
& \leq\left(\sup _{i} \widehat{\mu}_{\delta}\left(a_{i}\right)\right) \sum_{i=1}^{\infty} \int_{E_{\delta}\left(a_{i}\right)}|f|^{p} d V . \\
& \leq N\left(\sup _{i} \widehat{\mu}_{\delta}\left(a_{i}\right)\right) \int_{\Omega}|f|^{p} d V .
\end{aligned}
$$

Hence, $\mu$ is a Carleson measure on $b^{p}$. The proof is complete.

The above proof shows that the implications (c) $\Rightarrow(d) \Rightarrow$ (a) still hold for $p=1$. So, we have the following, which will be used in the characterization of bounded positive Toeplitz operators.

Corollary 3.6. If $\mu \geq 0$ is a Carleson measure, then $\mu$ is a Carleson measure on $b^{1}$.

Also, by carefully examining the proof above, one can see that the following equivalences between various quantities.

Corollary 3.7. Let $1<p<\infty$ and $\delta \in(0,1)$. For $\mu \geq 0$, we have

$$
\sup _{0 \neq f \in b^{p}} \frac{\int_{\Omega}|f|^{p} d \mu}{\int_{\Omega}|f|^{p} d V} \approx \sup _{a \in \Omega} \tilde{\mu}_{p}(a) \approx \sup _{a \in \Omega} \widehat{\mu}_{\delta}(a) \approx \sup _{i} \widehat{\mu}_{\delta}\left(a_{i}\right) .
$$

Having Theorem 3.5, we now turn to the characterizations of bounded positive Toeplitz operators on $b^{p}$. For $\mu \geq 0$, recall that the Toeplitz operator $T_{\mu}$ densely defined on $b^{p}$ is given by

$$
T_{\mu} f(x)=\int_{\Omega} R(x, y) f(y) d \mu(y)
$$


for functions $f \in b^{\infty}$.

Lemma 3.8. Let $\mu \geq 0$ be a Carleson measure. Then we have

$$
\left\langle T_{\mu} f, g\right\rangle=\int_{\Omega} f \bar{g} d \mu
$$

for $f, g \in b^{\infty}$.

Proof. Since $\mu$ is a Carleson measure on $b^{1}$ by Corollary 3.6, we have by Lemma 2.1

$$
\int_{\Omega}|R(x, y)| d \mu(y) \lesssim \int_{\Omega}|R(x, y)| d y \lesssim \int_{\Omega} d(x, y)^{-n} d y
$$

for $x \in \Omega$ and therefore

$$
\begin{aligned}
\int_{\Omega} \int_{\Omega}|R(x, y)| d \mu(y) d x & \lesssim \int_{\Omega} \int_{\Omega} d(x, y)^{-n} d y d x \\
& \lesssim\left[\int_{\Omega}\left(\int_{\Omega} d(x, y)^{-n} d y\right)^{2} d x\right]^{1 / 2} .
\end{aligned}
$$

In the meantime, by Lemma 2.2 , we have

$$
\begin{aligned}
\left(\int_{\Omega} d(x, y)^{-n} d y\right)^{2} & =\left(\int_{\Omega} \frac{r(y)^{1 / 4}}{d(x, y)^{n / 2}} \cdot \frac{1}{d(x, y)^{n / 2} r(y)^{1 / 4}} d y\right)^{2} \\
& \leq\left(\int_{\Omega} \frac{r(y)^{1 / 2}}{d(x, y)^{n}} d y\right)\left(\int_{\Omega} \frac{1}{d(x, y)^{n} r(y)^{1 / 2}} d y\right) \\
& \lesssim \frac{1}{r(x)^{1 / 2}} \int_{\Omega} \frac{r(y)^{1 / 2}}{d(x, y)^{n}} d y
\end{aligned}
$$

for $x \in \Omega$. It follows from Fubini's theorem and Lemma 2.2 again that

$$
\int_{\Omega} \int_{\Omega}|R(x, y)| d \mu(y) d x \lesssim \int_{\Omega} \int_{\Omega} \frac{r(y)^{1 / 2}}{d(x, y)^{n} r(x)^{1 / 2}} d x d y<\infty .
$$

This justifies interchanging the order of integrations below. Now, for $f, g \in$ $b^{\infty}$, we have

$$
\begin{aligned}
<T_{\mu} f, g> & =\int_{\Omega} \overline{g(x)} \int_{\Omega} R(x, y) f(y) d \mu(y) d x \\
& =\int_{\Omega} f(y) \int_{\Omega} R(x, y) \overline{g(x)} d x d \mu(y) \\
& =\int_{\Omega} f(y) \overline{g(y)} d \mu(y) .
\end{aligned}
$$


The proof is complete.

Now, we characterize positive bounded Toeplitz operators.

TheOrem 3.9. Let $\mu \geq 0$ and $1<p<\infty$. Then, the following two conditions are equivalent.

(a) $T_{\mu}: b^{p} \rightarrow b^{p}$ is bounded.

(b) $\mu$ is a Carleson measure.

Moreover, $\left\|T_{\mu}\right\|$ is equivalent to any of quantities in Corollary 3.7.

Proof. First assume that $T_{\mu}$ is bounded on $b^{p}$. Let $a \in \Omega$. By Proposition 8.1 of [1] we have

$$
\left|T_{\mu} k_{a, p}(a)\right| \lesssim r(a)^{-\frac{n}{p}}\left\|T_{\mu} k_{a, p}\right\|_{p} \leq r(a)^{-\frac{n}{p}}\left\|T_{\mu}\right\| .
$$

On the other hand, by Lemma 2.4, we have

$$
\left|T_{\mu} k_{a, p}(a)\right| \approx r(a)^{n\left(1-\frac{1}{p}\right)} \int_{\Omega}|R(a, y)|^{2} d \mu(y) .
$$

Combining these two, we have

$$
r(a)^{n} \int_{\Omega}|R(a, y)|^{2} d \mu(y) \lesssim\left\|T_{\mu}\right\| .
$$

Now, let $\delta=\delta_{0}$ where $\delta_{0}$ is the number provided by Lemma 2.3. Then, we have by Lemma 2.3 and (3.3)

$$
\widehat{\mu}_{\delta}(a) \approx \frac{\mu\left(E_{\delta}(a)\right)}{r(a)^{n}} \approx r(a)^{n} \int_{E_{\delta}(a)}|R(a, y)|^{2} d \mu(y) \lesssim\left\|T_{\mu}\right\| .
$$

This is true for all $a \in \Omega$ and constants abbreviated above are independent of $a$. Thus, we have $\sup _{a \in \Omega} \widehat{\mu}_{\delta}(a) \lesssim\left\|T_{\mu}\right\|$ and thus $\mu$ is a Carleson measure by Theorem 3.5.

Now, suppose that $\mu$ is a Carleson measure. Let $f, g \in b^{\infty}(\Omega)$. By Lemma 3.8 and Corollary 3.7, we have

$$
\begin{aligned}
\left|<T_{\mu} f, g>\right| & =\left|\int_{\Omega} f \bar{g} d \mu\right| \\
& \leq\left(\int_{\Omega}|f|^{p} d \mu\right)^{1 / p}\left(\int_{\Omega}|g|^{p^{\prime}} d \mu\right)^{1 / p^{\prime}} \\
& \lesssim \sup _{a \in \Omega} \widehat{\mu}_{\delta}(a)\left(\int_{\Omega}|f|^{p} d V\right)^{1 / p}\left(\int_{\Omega}|g|^{p^{\prime}} d V\right)^{1 / p^{\prime}}
\end{aligned}
$$


where the last inequality holds, because $\mu$ is a Carleson measure. Since $b^{\infty}$ is dense in both $b^{p}$ and $b^{p^{\prime}}$ by Lemma 2.5, a duality argument using Lemma 2.6 shows that $T_{\mu}$ is bounded on $b^{p}$ and $\left\|T_{\mu}\right\| \lesssim \sup _{a \in \Omega} \widehat{\mu}_{\delta}(a)$. The proof is complete.

Next, we give the corresponding characterization for compact positive Toeplitz operators. In order to do so, we need the notion of vanishing Carleson measures. For $\mu \geq 0$ and $1<p<\infty$, we say that $\mu$ is a vanishing Carleson measure on $b^{p}$ if the inclusion $i_{p}: b^{p} \rightarrow L^{p}(\mu)$ is compact, or equivalently, if

$$
\int_{\Omega}\left|f_{k}\right|^{p} d \mu \rightarrow 0
$$

whenever $f_{k} \rightarrow 0$ weakly in $b^{p}$. To characterize vanishing Carleson measures on $b^{p}$, we first need the following.

Lemma 3.10. Let $1<p<\infty$. Then $k_{a, p} \rightarrow 0$ weakly in $b^{p}$ as $a \rightarrow \partial \Omega$.

Proof. Let $f \in b^{p^{\prime}}$. Using Lemma 2.4, we have

$$
\left|\left\langle k_{a, p}, f\right\rangle\right| \approx r(a)^{n\left(1-\frac{1}{p}\right)}|f(a)| \rightarrow 0
$$

as $a \rightarrow \partial \Omega$. The proof is complete.

Now, we have a characterization for vanishing Carleson measures.

Theorem 3.11. Let $1<p<\infty$ and $\delta \in(0,1)$. For $\mu \geq 0$, the following conditions are all equivalent.

(a) $\mu$ is a vanishing Carleson measure on $b^{p}$.

(b) $\lim _{a \rightarrow \partial \Omega} \tilde{\mu}_{p}(a)=0$.

(c) $\lim _{a \rightarrow \partial \Omega} \widehat{\mu}_{\delta}(a)=0$.

(d) $\lim _{i \rightarrow \infty} \widehat{\mu}_{\delta}\left(a_{i}\right)=0$.

One can see from the theorem above that the notion of vanishing Carleson measures on $b^{p}$ is also independent of $1<p<\infty$. So, we will simply say that $\mu \geq 0$ is a vanishing Carleson measure if one of the four conditions above holds for $\mu$. 
Proof. Since $k_{a, p} \rightarrow 0$ weakly in $b^{p}$ as $a \rightarrow \partial \Omega$ by Lemma 3.10, we clearly have $(\mathrm{a}) \Rightarrow(\mathrm{b})$.

Next, we assume (b) and show (c). By (3.2) we have

$$
\widehat{\mu}_{\delta}(a) \lesssim \int_{\Omega}\left|k_{a, p}\right|^{p} d \mu=\tilde{\mu}_{p}(a) \rightarrow 0 \quad(a \rightarrow \partial \Omega)
$$

for $\delta=\delta_{0}$ where $\delta_{0}$ is the number provided by Lemma 2.3. Hence, (c) holds for a small $\delta$. But, an application of Lemma 3.2 shows that (c) holds in fact for all $\delta$.

Since $a_{i} \rightarrow \partial \Omega$, we have (c) $\Rightarrow$ (d).

Finally, we assume (d) and show (a). Let $\left\{f_{k}\right\}$ be a sequence converging to 0 weakly in $b^{p}$. By the proof of (d) $\Rightarrow$ (a) of Theorem 3.5, we have

$$
\int_{\Omega}\left|f_{k}\right|^{p} d \mu \lesssim \sum_{i<j} \int_{E_{\delta / 3}\left(a_{i}\right)}\left|f_{k}\right|^{p} d V+N \sup _{i \geq j} \widehat{\mu}_{\delta}\left(a_{i}\right) \int_{\Omega}\left|f_{k}\right|^{p} d V
$$

for any $i, j$. Here, $N$ is the positive integer provided by Lemma 3.4. Since $f_{k} \rightarrow 0$ weakly in $b^{p}$, one can easily see that $f_{k} \rightarrow 0$ uniformly on compact subsets of $\Omega$ and $\left\{f_{k}\right\}$ is bounded in $L^{p}$-norm. Thus, fixing $j$ and taking the limit $k \rightarrow \infty$ in (3.4), we obtain

$$
\limsup _{k} \int_{\Omega}\left|f_{k}\right|^{p} d \mu \lesssim \sup _{i \geq j} \widehat{\mu}_{\delta}\left(a_{i}\right)
$$

for each $j$. Note that we have by assumption $\sup _{i \geq j} \widehat{\mu}_{\delta}\left(a_{i}\right) \rightarrow 0$ as $j \rightarrow \infty$. Thus, taking the limit $j \rightarrow \infty$, we conclude

$$
\underset{k}{\limsup } \int_{\Omega}\left|f_{k}\right|^{p} d \mu=0 .
$$

Namely, $\mu$ is a vanishing Carleson measure, as desired. The proof is complete.

As a result corresponding to Theorem 3.9, we characterize compact positive Toeplitz operators in terms of vanishing Carleson measures.

Theorem 3.12. Let $\mu \geq 0$ and $1<p<\infty$. Then, the following two conditions are equivalent.

(a) $T_{\mu}: b^{p} \rightarrow b^{p}$ is compact.

(b) $\mu$ is a vanishing Carleson measure. 
Proof. First, suppose that $T_{\mu}$ is compact. By Lemma 2.4, we have

$$
\left|<T_{\mu} k_{a, p}, k_{a, p^{\prime}}>\right| \approx r(a)^{n} \int_{\Omega}|R(a, y)|^{2} d \mu(y) \approx \tilde{\mu}_{2}(a)
$$

for all $a \in \Omega$. On the other hand, since $k_{a, p} \rightarrow 0$ weakly in $b^{p}$ as $a \rightarrow \partial \Omega$ by Lemma 3.10, Hölder's inequality and compactness of $T_{\mu}$ yield

$$
\left|<T_{\mu} k_{a, p}, k_{a, p^{\prime}}>\right| \leq\left\|T_{\mu} k_{a, p}\right\|_{p} \rightarrow 0 \quad(a \rightarrow \partial \Omega) .
$$

Thus, by (3.5), (3.6) and Theorem 3.11, we conclude that $\mu$ is a vanishing Carleson measure.

Now, suppose that $\mu$ is a vanishing Carleson measure. For $\epsilon>0$, let $\mu_{\epsilon}=\chi_{\Omega_{\epsilon}} d \mu$ where $\Omega_{\epsilon}=\{x \in \Omega: r(x) \geq \epsilon\}$. Since each $\mu_{\epsilon}$ is supported on a compact subset of $\Omega$, one can easily verify that the corresponding Toeplitz operator $T_{\mu_{\epsilon}}$ is compact. Hence, in order to show the compactness of $T_{\mu}$, it is sufficient to show that $T_{\mu_{\epsilon}} \rightarrow T_{\mu}$ in the operator norm.

Let $\tau_{\epsilon}=\mu-\mu_{\epsilon}$. Fix $\delta \in(0,1)$. Note that if $a \in \Omega$ and $r(a) \geq \frac{\epsilon}{1-\delta}$, then $E_{\delta}(a) \subset \Omega_{\epsilon}$ by Lemma 3.1. Therefore, we have

$$
\sup _{a \in \Omega} \widehat{\left(\tau_{\epsilon}\right)_{\delta}}(a) \leq \sup _{r(a)<\frac{\epsilon}{1-\delta}} \widehat{\mu}_{\delta}(a) \rightarrow 0 \quad(\epsilon \rightarrow 0)
$$

where the convergence holds by Theorem 3.11. Now, since $T_{\mu}-T_{\mu_{\epsilon}}=T_{\tau_{\epsilon}}$, we conclude by Theorem 3.9

$$
\left\|T_{\mu}-T_{\mu_{\epsilon}}\right\| \approx \sup _{a \in \Omega} \widehat{\left(\tau_{\epsilon}\right)_{\delta}}(a) \rightarrow 0 \quad(\epsilon \rightarrow 0)
$$

as desired. The proof is complete.

We now turn to the characterization of positive Schatten class Toeplitz operators. Before proceeding, let's review briefly some basic facts about Schatten class operators.

For a compact operator $T$ on a separable Hilbert space $X$, let $\left\{s_{m}(T)\right\}$ be the nonzero eigenvalues (listed by multiplicity) of $|T|=\left(T^{*} T\right)^{1 / 2}$ arranged so that the sequence is non-increasing, where $T^{*}$ denotes the Hilbert space adjoint of $T$. This sequence is called the singular value sequence of $T$. For $1 \leq p<\infty$, we say $T$ is a Schatten $p$-class operator if

$$
\|T\|_{S_{p}(X)}=\left(\sum_{m}\left|s_{m}(T)\right|^{p}\right)^{1 / p}<\infty .
$$


Let $S_{p}(X)$ be the space of all Schatten $p$-class operators on $X$. As is well known, $S_{p}(X)$ is a Banach space with the above norm and is a two-sided ideal in the space of all bounded linear operators on $X$.

Also, for $T \in S_{1}(X)$ and an orthonormal basis $\left\{e_{m}\right\}$ for $X$, the sum

$$
\operatorname{tr}(T)=\sum_{m}\left\langle T e_{m}, e_{m}\right\rangle
$$

is absolutely convergent and independent of the choice of $\left\{e_{m}\right\}$. The sum above is called the trace of $T$. If $T \in S_{p}(X)$ and $T \geq 0$, we have

$$
\|T\|_{S_{p}(X)}=\left[\operatorname{tr}\left(T^{p}\right)\right]^{1 / p}
$$

for $1 \leq p<\infty$. See [9], for example, for more information and related facts.

In the rest of this section we use the notations $S_{p}=S_{p}\left(b^{2}\right), \tilde{\mu}=\tilde{\mu}_{2}$ and $k_{x}=k_{x, 2}$ for simplicity. Also, the measure $d \lambda$ is defined on $\Omega$ by

$$
d \lambda(x)=R(x, x) d V(x) .
$$

Note that $\|R(x, \cdot)\|_{2}^{2}=R(x, x)$. Hence, using the same arguments of Lemma 13 in [10], we have

$$
\operatorname{tr}(T)=\int_{\Omega}\langle T R(x, \cdot), R(x, \cdot)\rangle d x=\int_{\Omega}\left\langle T k_{x}, k_{x}\right\rangle d \lambda(x)
$$

for every $T \in S_{1}$.

We now give a characterization of Schatten class positive Toeplitz operators on $b^{2}(\Omega)$.

Theorem 3.13. Let $1 \leq p<\infty$ and $\delta \in(0,1)$. For $\mu \geq 0$, the following conditions are all equivalent.

(a) $T_{\mu} \in S_{p}$.

(b) $\tilde{\mu} \in L^{p}(\lambda)$.

(c) $\widehat{\mu}_{\delta} \in L^{p}(\lambda)$.

(d) $\sum_{i} \widehat{\mu}_{\delta}\left(a_{i}\right)^{p}<\infty$.

Moreover, we have

$$
\|\left. T_{\mu}\right|_{S_{p}} \approx\left(\int_{\Omega}|\tilde{\mu}|^{p} d \lambda\right)^{1 / p} \approx\left(\int_{\Omega}\left|\widehat{\mu}_{\delta}\right|^{p} d \lambda\right)^{1 / p} \approx\left(\sum_{i} \widehat{\mu}_{\delta}\left(a_{i}\right)^{p}\right)^{1 / p} .
$$


Proof. First, suppose (a) and show (b). Since $T_{\mu} \geq 0$, it follows from (3.7) that

$$
\left\|T_{\mu}\right\|_{S_{p}}^{p}=\operatorname{tr}\left(T_{\mu}^{p}\right)=\int_{\Omega}\left\langle T_{\mu}^{p} k_{x}, k_{x}\right\rangle d \lambda(x) .
$$

It follows from Proposition 6.3.3 of [9] and Lemma 3.8 that

$$
\left\|T_{\mu}\right\|_{S_{p}}^{p} \geq \int_{\Omega}\left\langle T_{\mu} k_{x}, k_{x}\right\rangle^{p} d \lambda(x)=\int_{\Omega} \tilde{\mu}(x)^{p} d \lambda(x),
$$

so we have (b).

Next, suppose (b) and show (c). Fix $\delta=\delta_{0}$ where $\delta_{0}$ is the number provided by Lemma 2.3. By Lemma 2.1 and Lemma 2.4, we have

$$
\begin{aligned}
\int_{\Omega} \tilde{\mu}(x)^{p} d \lambda(x) & \geq \int_{\Omega}\left(\int_{E_{\delta}(x)}\left|k_{x}\right|^{2} d \mu\right)^{p} d \lambda(x) \\
& =\int_{\Omega}\left(R(x, x)^{-1} \int_{E_{\delta}(x)}|R(x, y)|^{2} d \mu(y)\right)^{p} d \lambda(x) \\
& \approx \int_{\Omega} \widehat{\mu}_{\delta}(x)^{p} d \lambda(x)
\end{aligned}
$$

So, we have (c) for a small $\delta$. But, a simple application of Lemma 3.2 shows that (c) holds for all $\delta$.

Now, suppose (c) and show (d). By Lemma 3.2 and Jensen's inequality, we have

$$
\widehat{\mu}_{\delta}\left(a_{i}\right)^{p} \lesssim \frac{1}{r\left(a_{i}\right)^{n}} \int_{E_{\delta}\left(a_{i}\right)} \widehat{\mu}_{\delta}(x)^{p} d x \approx \int_{E_{\delta}\left(a_{i}\right)} \widehat{\mu}_{\delta}(x)^{p} d \lambda(x)
$$

for all $i$. Summing up all these together, we have

$$
\sum_{i} \widehat{\mu}_{\delta}\left(a_{i}\right)^{p} \lesssim \sum_{i} \int_{E_{\delta}\left(a_{i}\right)} \widehat{\mu}_{\delta}(x)^{p} d \lambda(x) \lesssim N \int_{\Omega} \widehat{\mu}_{\delta}(x)^{p} d \lambda(x)<\infty
$$

where $N$ is the positive integer provided by Lemma 3.4. Thus, we have (d).

Finally, suppose (d) and show (a). First, consider the case $p=1$. By (3.7) and Lemma 3.8, we have

$$
\operatorname{tr}\left(T_{\mu}\right)=\int_{\Omega} \int_{\Omega}|R(x, y)|^{2} d \mu(y) d x=\int_{\Omega}|R(y, y)|^{2} d \mu(y)
$$

Thus, by Lemma 2.1 and Lemma 3.1, we have

$$
\operatorname{tr}\left(T_{\mu}\right) \leq \sum_{i} \int_{E_{\delta}\left(a_{i}\right)} R(y, y) d \mu(y) \approx \sum_{i} \widehat{\mu}_{\delta}\left(a_{i}\right)<\infty .
$$


So, we have $T_{\mu} \in S_{1}$. Now, consider the case $1<p<\infty$. For any $\zeta \in \mathbb{C}$ with $0 \leq \operatorname{Re} \zeta \leq 1$, define a complex Borel measure $\mu_{\zeta}$ on $\Omega$ by

$$
d \mu_{\zeta}(x)=\sum_{i}\left[\widehat{\mu}_{\delta}\left(a_{i}\right)\right]^{p \zeta-1} \chi_{E_{\delta}\left(a_{i}\right)}(x) d \mu(x)
$$

and consider corresponding Toeplitz operators $T_{\mu_{\zeta}}$ acting on $b^{2}$. Note $T_{\mu} \leq$ $T_{\mu_{\frac{1}{p}}}$. Thus, the complex interpolation (see Theorem 2.2.7 of [9]) gives

$$
\left\|T_{\mu}\right\|_{S_{p}} \leq\left\|T_{\mu_{\frac{1}{p}}}\right\|_{S_{p}} \leq M_{0}^{1-\frac{1}{p}} M_{1}^{\frac{1}{p}},
$$

where $M_{0}=\sup \left\{\left\|T_{\mu_{\zeta}}\right\|: \operatorname{Re} \zeta=0\right\}$ and $M_{1}=\sup \left\{\left\|T_{\mu_{\zeta}}\right\|_{S_{1}}: \operatorname{Re} \zeta=1\right\}$. One can see $M_{0}<\infty$ by the same argument as in the proof of Theorem 12 in [10]. To estimate $M_{1}$, let $\left\{f_{k}\right\}$ and $\left\{g_{k}\right\}$ be two orthonormal bases for $b^{2}$. Then, for $\operatorname{Re} \zeta=1$, it follows from the same way as in the proof of Theorem 12 of [10] that

$$
\sum_{k}\left|<T_{\mu_{\zeta}} f_{k}, g_{k}>\right| \leq \sum_{k} \int_{\Omega}\left|f_{k}\right|\left|g_{k}\right| d\left|\mu_{\zeta}\right| \leq \int_{\Omega} R(x, x) d\left|\mu_{\zeta}\right|(x)
$$

and thus

$$
\left\|T_{\mu_{\zeta}}\right\|_{S_{1}} \lesssim \sum_{k}\left[\widehat{\mu}_{\delta}\left(a_{i}\right)\right]^{p-1} \int_{E_{\delta}\left(a_{i}\right)} R(x, x) d \mu(x) \approx \sum_{k} \widehat{\mu}_{\delta}\left(a_{i}\right)^{p} .
$$

So, we have

$$
\left\|T_{\mu}\right\|_{S_{p}}^{p} \lesssim M_{1} \lesssim \sum_{i}\left(\widehat{\mu}_{\delta}\left(a_{i}\right)\right)^{p}<\infty
$$

This completes the proof.

\section{§4. Toeplitz operators with continuous symbols}

In this section, we consider uniformly continuous symbols. That is, we consider symbols continuous up to the boundary and then describe the essential spectra of corresponding Toeplitz operators.

Let's recall the notion of the essential spectrum. Let $\mathcal{B}$ be the algebra of all bounded linear operators on a Banach space $X$ and $\mathcal{K}$ be the two-sided compact ideal of $\mathcal{B}$. For an operator $T \in \mathcal{B}$ and a complex number $\eta$, we say that $\eta \in \sigma_{e}(T ; X)$, the essential spectrum of $T$, if $(T-\eta)+\mathcal{K}$ is not invertible in the Calkin algebra $\mathcal{B} / \mathcal{K}$. In other words, $\eta \in \sigma_{e}(T ; X)$ if and only if $T-\eta$ is not Fredholm. 
We need compactness of Hankel operators with symbols continuous up to the boundary. For $\varphi \in L^{\infty}$, the Hankel operator $H_{\varphi}$ with symbol $\varphi$ is defined by

$$
H_{\varphi} f=\varphi f-Q(\varphi f)
$$

for $f \in b^{1}$. Let $1<p<\infty$. Then, since $Q$ is bounded on $L^{p}$, it is clear that $H_{\varphi}: b^{p} \rightarrow L^{p}$ is also bounded and $\left\|H_{\varphi}\right\| \lesssim\|\varphi\|_{L^{\infty}}$. The integral representation of $H_{\varphi}$ is given by

$$
H_{\varphi} f(x)=\int_{\Omega} R(x, y)[\varphi(x)-\varphi(y)] f(y) d y \quad(x \in \Omega)
$$

for $f \in b^{p}$.

LEMMA 4.1. Let $1<p<\infty$ and $K$ be a measurable function on $\Omega \times \Omega$ for which

$$
\sup _{x \in \Omega} \int_{\Omega}|K(x, y)|^{p^{\prime}} d y<\infty .
$$

Then, the integral operator $T$ defined by

$$
T h(x)=\int_{\Omega} K(x, y) h(y) d y \quad(x \in \Omega)
$$

is a compact operator from $L^{p}$ into $L^{p}$.

Proof. See Exercise VI.53 of [3].

Theorem 4.2. Let $1<p<\infty$ and $\varphi \in C(\bar{\Omega})$. Then, $H_{\varphi}: b^{p} \rightarrow L^{p}$ is compact.

Proof. Fix $1<p<\infty$ and let $M_{p}$ be the set of all functions $\varphi \in C(\bar{\Omega})$ for which the corresponding Hankel operators $H_{\varphi}: b^{p} \rightarrow L^{p}$ are compact. We need to show $M_{p}=C(\bar{\Omega})$.

First, we show that $M_{p}$ is a closed subalgebra of $C(\bar{\Omega})$. Let $\left\{\psi_{k}\right\}$ be a sequence in $M_{p}$ converging to $\psi$ in $C(\bar{\Omega})$. Then, we have

$$
\left\|H_{\psi_{k}}-H_{\psi}\right\|=\left\|H_{\psi_{k}-\psi}\right\| \lesssim\left\|\psi_{k}-\psi\right\|_{L^{\infty}} \rightarrow 0 .
$$

It follows that $H_{\psi}$ is compact, because each $H_{\psi_{k}}$ is compact. Hence, $M_{p}$ is a closed subspace of $C(\bar{\Omega})$. Now, for any $\varphi, \psi \in L^{\infty}$, a straightforward calculation yields

$$
H_{\varphi \psi}=\widetilde{H}_{\varphi} H_{\psi}+H_{\varphi} T_{\psi}
$$


where $\widetilde{H}_{\varphi}$ denotes the "Hankel" operator extended to the whole $L^{p}$. This shows that $M_{p}$ is an algebra.

Next, we show that $M_{p}$ contains all the polynomials. So, let $\varphi$ be a polynomial and consider an integral operator $T$ defined by

$$
T f(x)=\int_{\Omega} R(x, y)[\varphi(x)-\varphi(y)] f(y) d y \quad(x \in \Omega)
$$

for $f \in L^{1}$. Note that, when restricted to $b^{p}, T$ reduces to $H_{\varphi}$ acting on $b^{p}$. Since $T f=\varphi Q(f)-Q(\varphi f), T$ clearly is bounded on $L^{q}$ for all $1<q<\infty$. We claim that $T: L^{q} \rightarrow L^{q}$ is compact for $q>n$. So, let $q>n$. Note that $|\varphi(x)-\varphi(y)| \lesssim|x-y|$ for $x, y \in \Omega$. Thus, by Lemma 4.1, it is sufficient to show that

$$
\sup _{x \in \Omega} \int_{\Omega}|x-y|^{q^{\prime}}|R(x, y)|^{q^{\prime}} d y<\infty .
$$

By Lemma 2.1, we have

$$
|x-y||R(x, y)| \lesssim|x-y| d(x, y)^{-n} \leq|x-y|^{1-n}
$$

for $x, y \in \Omega$. Note $q^{\prime}(1-n)>-n$. Thus, taking $c>0$ so that $\Omega \subset\{x \in$ $\left.\mathbb{R}^{n}:|x| \leq c\right\}$, we obtain

$$
\sup _{x \in \Omega} \int_{\Omega}|x-y|^{q^{\prime}(1-n)} d y \lesssim \int_{|y| \leq 2 c}|y|^{q^{\prime}(1-n)} d y<\infty
$$

and thus (4.1) holds. Having seen that $T: L^{q} \rightarrow L^{q}$ is compact whenever $q>n$, one may now repeat the same interpolation argument as in the proof of Theorem 4.4 in [4] to see that $T: L^{p} \rightarrow L^{p}$, as well as $H_{\varphi}=\left.T\right|_{b^{p}}: b^{p} \rightarrow$ $L^{p}$, is compact. Thus, $\varphi \in M_{p}$.

Now, since $M_{p}$ is a closed subalgebra of $C(\bar{\Omega})$ containing all the polynomials, we conclude $M_{p}=C(\bar{\Omega})$ by the Stone-Weierstrass theorem. The proof is complete.

As a consequence of Theorem 4.2, we have the following which will be used in the proof of Theorem 4.5 below.

Corollary 4.3. Let $1<p<\infty$. If $\varphi, \psi \in C(\bar{\Omega})$, then both $T_{\varphi \psi}-$ $T_{\varphi} T_{\psi}$ and $T_{\varphi} T_{\psi}-T_{\psi} T_{\varphi}$ are compact on $b^{p}$. 
Proof. For any $\varphi, \psi \in L^{\infty}$, a straightforward calculation yields

$$
T_{\varphi \psi}=T_{\varphi} T_{\psi}+\widetilde{T}_{\varphi} H_{\psi}
$$

where $\widetilde{T}_{\varphi}$ denotes the "Toeplitz" operator extended to the whole $L^{p}$. This, together with Theorem 4.2, shows the compactness of $T_{\varphi \psi}-T_{\varphi} T_{\psi}$ on $b^{p}$ for $\psi \in C(\bar{\Omega})$. Hence, $T_{\varphi} T_{\psi}-T_{\psi} T_{\varphi}$ is compact on $b^{p}$ for $\varphi, \psi \in C(\bar{\Omega})$.

Also, we need the following (we shall see that the converse is also true).

LemmA 4.4. Let $1<p<\infty$ and $\varphi \in C(\bar{\Omega})$. If $\varphi=0$ on $\partial \Omega$, then $T_{\varphi}$ is compact on $b^{p}$.

Proof. Without loss of generality assume $\varphi \geq 0$. Since $\varphi=0$ on $\partial \Omega$, there exists a sequence $\left\{\varphi_{k}\right\} \subset C(\bar{\Omega})$ with compact support in $\Omega$ such that $\varphi_{k} \geq 0$ and $\varphi_{k} \rightarrow \varphi$ in $L^{\infty}$. So, $T_{\varphi_{k}} \rightarrow T_{\varphi}$ in the operator norm. Since each $T_{\varphi_{k}}$ is compact on $b^{p}$ by Theorem 3.11 , we conclude that $T_{\varphi}$ is also compact. This completes the proof.

We now turn to the characterization of $\sigma_{e}\left(T_{\varphi} ; b^{p}\right)$ for $1<p<\infty$ and $\varphi \in C(\bar{\Omega})$.

TheOREm 4.5. Let $1<p<\infty$ and $\varphi \in C(\bar{\Omega})$. Then $\sigma_{e}\left(T_{\varphi} ; b^{p}\right)=$ $\varphi(\partial \Omega)$.

Proof. Fix $p$. First, we show $\varphi(\partial \Omega) \subset \sigma_{e}\left(T_{\varphi} ; b^{p}\right)$. Let $\eta \in \varphi(\partial \Omega)$ and assume $\eta=\varphi(\zeta)$ for some $\zeta \in \partial \Omega$. For simplicity, assume $\eta=0$; consider otherwise $\varphi-\eta$. Suppose that $T_{\varphi}$ is Fredholm on $b^{p}$. Then, there exists a bounded linear operator $S$ on $b^{p}$ such that $S T_{\varphi}-I$ is compact on $b^{p}$. Since $k_{x, p} \rightarrow 0$ weakly in $b^{p}$ as $x \rightarrow \partial \Omega$ by Lemma 3.10, we have $\left\|S T_{\varphi} k_{x, p}-k_{x, p}\right\|_{p} \rightarrow 0$ as $x \rightarrow \partial \Omega$. In particular, since each $k_{x, p}$ has $L^{p}$-norm 1, we have

$$
\left\|S T_{\varphi}\left(k_{x, p}\right)\right\|_{p} \rightarrow 1
$$

as $x \rightarrow \zeta$.

On the other hand, by Lemma 2.1 and Lemma 2.4, we have

$$
\begin{aligned}
\left\|S T_{\varphi} k_{x, p}\right\|_{p}^{p} & =\left\|S Q\left(\varphi k_{x, p}\right)\right\|_{p}^{p} \\
& \lesssim \int_{\Omega}|\varphi(y)|^{p} \frac{|R(x, y)|^{p}}{\|R(x, \cdot)\|_{p}^{p}} d y \\
& \lesssim M_{\delta}+\|\varphi\|_{\infty} r(x)^{n(p-1)} \int_{|y-\zeta| \geq \delta} \frac{1}{|x-y|^{p n}} d y
\end{aligned}
$$


where

$$
M_{\delta}=\sup \left\{|\varphi(y)|^{p}:|y-\zeta|<\delta, y \in \Omega\right\}
$$

for $\delta>0$. Thus, taking the limit $x \rightarrow \zeta$, we have

$$
\limsup _{x \rightarrow \zeta}\left\|S T_{\varphi}\left(k_{x, p}\right)\right\|_{p}^{p} \lesssim M_{\delta}
$$

for each $\delta>0$. Note that the constant abbreviated in the inequity above is independent of $\delta$. Since $\varphi \in C(\bar{\Omega}), M_{\delta} \rightarrow 0$ as $\delta \rightarrow 0$. Now, take $\delta \rightarrow 0$ to get $\left\|S T_{\varphi}\left(k_{x, p}\right)\right\|_{p} \rightarrow 0$ as $x \rightarrow \zeta$, which is a contradiction to (4.2).

Next, we show $\sigma_{e}\left(T_{\varphi} ; b^{p}\right) \subset \varphi(\partial \Omega)$. Let $\eta \notin \varphi(\partial \Omega)$ and assume $\eta=0$, as above. We need to show that $T_{\varphi}$ is Fredholm on $b^{p}$. Take $\psi \in C(\bar{\Omega})$ such that $\varphi \psi=1$ on $\partial \Omega$. Then, $T_{1-\varphi \psi}$ is compact on $b^{p}$ by Lemma 4.4. Note

$$
T_{\psi} T_{\varphi}-I=T_{\psi} T_{\varphi}-T_{\psi \varphi}-T_{1-\varphi \psi}
$$

It follows from Corollary 4.3 that $T_{\psi} T_{\varphi}-I$ is compact on $b^{p}$. Similarly, we see $T_{\varphi} T_{\psi}-I$ is compact on $b^{p}$. So, $T_{\varphi}$ is Fredholm on $b^{p}$, as desired. The proof is complete.

As an immediate application, we have the following.

Corollary 4.6. Let $1<p<\infty$ and $\varphi \in C(\bar{\Omega})$. Then, $T_{\varphi}$ is Fredholm on $b^{p}$ if and only if $\varphi$ is bounded away from 0 near $\partial \Omega$.

Let $\left\|T_{\varphi}\right\|_{p, e}$ denote the norm of $T_{\varphi}: b^{p} \rightarrow b^{p}$ in the Calkin algebra of $b^{p}$. Then, we also have the following as another consequence.

Corollary 4.7. Let $1<p<\infty$ and $\varphi \in C(\bar{\Omega})$. Then, we have

$$
\|\varphi\|_{L^{\infty}(\partial \Omega)} \leq\left\|T_{\varphi}\right\|_{p, e}
$$

and the equality holds for $p=2$. In particular, $T_{\varphi}$ is compact on $b^{p}$ if and only if $\varphi=0$ on $\partial \Omega$.

Proof. The assertion (4.3) follows from Theorem 4.5 and the spectral radius formula. It is easily seen that $T_{\varphi}^{*}=T_{\bar{\varphi}}$. Thus, by Corollary 4.3 , we see that $T_{\varphi}$ is normal in the Calkin algebra of $b^{2}$. Hence, the equality holds in (4.3) for $p=2$. The second part of the corollary follows from (4.3) and Lemma 4.4. The proof is complete. 


\section{REFERENCES}

[1] S. Axler, P. Bourdon and W. Ramey, Harmonic function theory, Springer-Verlag, New York, 1992.

[2] B. R. Choe, H. Koo and H. Yi, Positive Toeplitz operators between the harmonic Bergman spaces, Potential Analysis, 17 (2002), 307-335.

[3] N. Dunford and J. T. Schwartz, Linear operators, Part I, Interscience Publishers, Inc., New York, 1958.

[4] M. Jovović, Compact Hankel operators on the harmonic Bergman spaces, Integr. Eqn. Oper. Theory, 22 (1995), 295-304.

[5] H. Kang and H, Koo, Estimates of the harmonic Bergman kernel on smooth domains, J. Funct. Anal., 185 (2001), 220-239.

[6] J. Miao, Toeplitz operators on harmonic Bergman spaces, Integr. Eqn. Oper. Theory, 27 (1997), 426-438.

[7] O. L. Oleinik, Embedding theorems for weighted classed of harmonic and analytic functions, J. Soviet Math., 9 (1978), 228-243.

[8] K. Stroethoff, Compact Toeplitz operators on weighted harmonic Bergman spaces, J. Austral. Math. Soc. (Series A), 64 (1998), 136-148.

[9] K. Zhu, Operator theory in function spaces, Marcel Dekker. New York and Basel, 1989.

[10] K. Zhu, Positive Toeplitz operators on weighted Bergman spaces of bounded symmetric domains, Operator Th., 20 (1988), 329-357.

Boo Rim Choe

Department of Mathematics

Korea University

Seoul 136-701

Korea

cbr@korea.ac.kr

Young Joo Lee

Department of Mathematics

Mokpo National University

Chonnam 534-729

Korea

yjlee@mokpo.ac.kr

Kyunguk $\mathrm{Na}$

Department of Mathematics

Korea University

Seoul 136-701

Korea

nakyunguk@korea.ac.kr 